FERMILAB-CONf-82/25-EXP

7000.000

\author{
CHARM AND BEAUTY PHOTOPRODUCTION AT FERMILAB* \\ Jeffrey A. Appel \\ April 1982
}

* Invited talk to the Europhysics Study Conference on Search for Charm, Beauty and Truth, Erice, Sicily, November 16, 1981. 


\author{
INV ITED TALK TO THE EUROPHYS ICS STUDY CONFERENCE ON \\ SEARCH FOR CHARM, BEAUTY AND TRUTH \\ Erice, Sicily, November 16, 1981 \\ Charm and Beauty Photoproduction at Fermilab \\ Jeffrey A. Appel
}

\title{
INTRODUCTION
}

\begin{abstract}
Four manifestations of charm have been observed in photoproduction at Fermilab so $f$ ar. These four are (1) multimuon indications of the total charm cross section and observations of (2) $\Psi$ and $\Psi^{\prime},(3) \cdot D^{\circ}$ and $D^{*}$ and (4) $\Lambda$. The relevent photoproduction experiments in the search for Charm at Fermilab are the broad band neutral beam experiments by a Col umbia-Fermilab-Illinois (CFI) collaboration, the Tagged Photon Beam experiment by the TPS collaboration ${ }^{2}$ and the muon beam experiment with an active iron target by the Berkeley-Fermilab-Princeton (BFP) collaboration. ${ }^{3}$
\end{abstract}

The photon beam experiments have similar forward multiparticle spectrometers. There are important differences among the two experiments, however. These include differences in the beams (hadron contamination, energy and flux) and detectors (solid angle acceptance of the forward spectrometers, target system instrumentation and trigger capability). Both experiments include a two-magnet forward spectrometer system with two Cerenkov counters for charged particle identification. Wire chamber systems are installed as $f$ ar upstream as possible allowing large angle acceptance for those particles which pass through the first magnet, but do not continue all the way through the downstream spectrometers. The Tagged Photon beam energies used during last year's run by the TPS collaboration range from 50 to $150 \mathrm{GeV}$ with no hadron contamination. The energy of each incident photon was known to a few percent. In the broad band neutral beam; the energy and intensities were higher. However, the $1 \%$ hadron contamination in the beam resulted in half the event triggers and serious backgrounds for some of the interesting physics. On the other hand, the average energy for observed charm events was typically around $150 \mathrm{GeV}$. In measuring $\Psi$ photoproduction, the Fermilab-IIlinois part of the CFI collaboration used liquid hydrogen and liquid deuterium targets while for the charmed particle production the full CFI group used a segmented scintillator target. The TPS experiment used a one and a half meter long liquid hydrogen target for all meas ur ements. 
In the TPS, the acceptance reaches out to approximately \pm 150 milliradians in the verticle and horizontal directions, while in the broad band neutral beam, acceptances are just a little more than half this size. In order to make use of the larger angular acceptance, the TPS cerenkov counters have 20 cells in each, both upstream and downstream detectors, while the CFI chambers had 12 and 16 cells respectively. The steel absorber in the CFI spectrometer is divided longitudinally to allow insertion of hodoscopes. These were used in identifying muons and crudely projecting them back to the target for trigger purposes. A typical event in the TPS is shown schematically in Figure 1. A rather clean multiparticle event is seen in the forward spectrometer and a single recoil is seen in the system of cylindrical proportional wire chambers and scintillators surrounding the target. This system allows identification of protons and measurement of the kinetic energy up to approximately $0.5 \mathrm{GeV}$. The only directly measured. photoproduction data presented here comes from the broad band neutral beam experiments. Results from the TPS collaboration should start appearing soon. The multimuon spectrometer of the BFP collaboration has been discussed in detail elsewhere $e^{4}$ and will not be reviewed here.

\section{CHARM TOTAL CROSS SECTION}

In the Berkeley-Fermilab-Princeton muon experiment, the measurements are extrapolated to zero in $q^{2}$ of the virtual photon. This procedure provides equivalent photoproduction results from the muon scattering experiment. In the BFP experiment, the muon beam strikes a magnetized active iron target and the relevent charmed photoproduction results are derived from multi-muon events. The muons, other than the $f$ ast forward beam sign muons, are assumed to come primarily from decays of Charm in the dense target. We leave the bulk of the discussion of the total cross section measurements to later talks at the conference. In summary, however, the measurements by the Berkeley-Fermilab-Princeton group show a slightly rising Charm production (approaching $1 \mu \mathrm{b}$ per nucleon) by photons as the energy rises. As the experimenters note, this rise does not saturate the $r$ ise in the total photon cross section.5

\section{CHARMONIUM}

The $\Psi$ and $\Psi^{\prime}$ measurements reported here were made by the Fermilab-Illinois part of the CFI group. Their $\Psi^{\prime}$ results are nw. The experimental observation of rather clean dimuon and dilectron signals centered at the appropriate mass for the $J / \Psi$ allows the group to make measurements as a function of energy. The energy is obtained from the observed $\Psi$ energy. Only two track events were used, thereby excluding only $5 \%$ of events seen with extra tracks. Additional multiparticle events were vetoed by wide angle counters just downstream of the target and $a$ requirement of $\leq 6$ forward particles in the trigger. In the 
diffractive events, the incident photon energy is equal to the dilepton energy. The assumptions used in arriving at the final results are given in Table $I$. The decay to electron pairs and

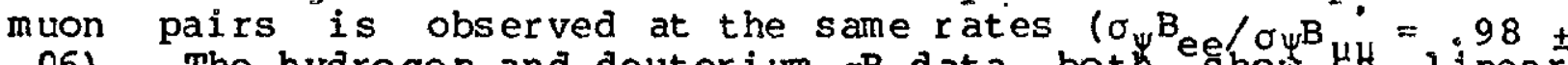
.06). The hydrogen and deuterium $\sigma B$ data both show $\mu_{\mathrm{H}} 1$ inear increase for photon energy between 60 and $300 \mathrm{GeV}$ with a slope of $(.007 \pm .0014) \mathrm{nb}$ per GeV. Most of the data is on deuterium. Yet enough hydrogen data was taken to see that except for the coherent deuterium peak near $t=0$, the $t$ slopes are consistent with each other. The deuterium data have $t$ shapes which are essentially energy independent.

The experimenters studied the character of the target recoil for the $95 \%$ of the $\Psi$ events which appeared to be diffractively produced as seen in the forward spectrometer. Two layers of scintillation counters and one layer of luci te Cerenkov counter were placed around the target for this purpose. As with photoproduction of rho's, the Fermilab-Illinois group found 708 of the events consistent with elastic scattering where a proton and only a proton appeared where it should or was absent where it shouldn't have gotten out of the target. These events are called elastic. The other $30 \%$ of the events, called quasi-elastic, either had additional hits or an extra particle in a non-coplaner recoil element. The elastic events were produced with do/dt proportional to $\exp (-2.8 t)$ while the quasi-elastic cross section was proportional to exp $(-1.3 t)$. All these results refer to the diffractively produced $\psi^{\prime} s$. The Berkeley-Fermilab-Princeton and EMC muon experiments report significant non-diffractive production of $\psi^{\prime} s$. They both observe a $\psi$ total cross section roughly consistent with that seen by the Fermilab-Illinois group. However, defining non-diffractive events (called inelastic by these groups) as those which have greater than $4-1 / 2$ to $5 \mathrm{GeV}$ of visible energy in the calorimeter target, the BFP and EMC groups see half the production as non-diffractive. 6

The Fermilab Illinois group sees $\psi^{\prime}$ to two muon and to two electron peaks at $3.68 \mathrm{GeV} / \mathrm{c}^{2}$ sitting on the tails of dileptons from the $\Psi$. An even cleaner sample of events consistent with the $\psi^{\prime}$ decaying to $\psi \pi \pi$ is shown in Figure 2. Notice that there are only a few events consistent with non-diffractive $\Psi$ production. The $\Psi^{\prime}$ results are summarized in Table II. Note that the $\psi^{\prime}$ photoproduction cross section at an aver age energy of $160 \mathrm{GeV}$ is about 208 of that of the $\Psi$ at a similar energy. More significantly, using the value $d_{\sigma} / d t$ at $t=0$ in a vector. dominance model, $\sigma_{y}$ 'p and $\sigma_{\psi p}$ are about the same size: This makes it reasonable to'p consider ${ }^{\sigma}$ he $\psi^{\prime}$, like the $\psi$, in the family of hadrons as required to consider them related to the open charm which is discussed next. 
We will concentrate here on the recent $D^{*}$ production data and then look at two unusual branching modes of the $D^{\circ}$.

In order to see the $D^{\circ}$, it is necessary to look at the $D^{\circ} \mathrm{s}$ which result from the decay of the $D^{*}$. Figure 3 shows two decay modes which have consistent and correct value $D^{\circ}$ mass peaks. In order to achieve these, a cut has been made on the Iow $D *-D$. mass difference. Figure 4 shows the enhancement at the appropriate $\Delta$ $\left(\Delta=M_{\mathrm{K} \pi \pi}-M_{\mathrm{K} \pi}\right)$ value in the $D^{*} \rightarrow D \pi$ decay for those events which have" the correct Do mass. It was by going back and cutting on this $\Delta$ value that gave the peaks in the previous figure.

The $D^{*}$ data are consistent with a pair production mechanism. Equal numbers of $\mathrm{D}^{*}$ and $\mathrm{D}^{*}$ - were seen in the experiment. The energy, $x, p_{t}$ and multiplicity distributions are all consistent with diffractive production. Furthermore, $35 \pm 98$ of the $D * ' s$ are produced with an additional charged $K$, consistent with an additional decaying D. From a study of the spectrum of $\pi$ ' $s$ produced with $D^{*} ' s$, the CFI group concludes that $45 \pm 25 \%$ of the $D^{*}$ 's are produced with another $D^{*}$. Both of these statistically poorly measured results are very suggestive of nearly pure diffractive production of the $D * ' s$.

Finally, the CFI collaboration reports results on the Cabbibo structure of $\mathrm{D}$ decays. They observe $\mathrm{D} \rightarrow \mathrm{K}^{+} \mathrm{K}^{-}$with branching fraction of $20 \pm 98$ (compared to the SPEAR result of $11 \pm 38$ ). The preaiction of simple Cabbibo theory is 4.68 . This collaboration, therefore, is not inconsistant with the surprisingly large value observed at SPEAR. Finally, the limit. on $D^{\circ} D^{\circ} \mathrm{mixing}$ (or doubly suppressed Cabbibo decay mode of irregular charge combinations of $K \pi$ ) gives a branching fraction limit of less than 118 at the 908 confidence level (compared to the SPEAR result of $(16 \%)$.

\section{CHARMED BARYONS}

Average properties of the $\Lambda_{c}$ events observed by the CFI group are listed in Table III. CThe properties they observed for the charmed baryons are 1 isted in Table IV. Notice the approximately equal numbers of both charge states in the decay modes $\mathrm{pk}$ and $\overline{\mathrm{p}} \mathrm{K}$, of $\mathrm{Figure} \mathrm{V}$. This again suggests diffractive pair production.

Limits on other decay modes of the $\Lambda_{c}$ are given in Table $V$ as derived from the data shown in Figure ${ }^{c} 6$. The shaded areas in Figure 6 represent the events produced by the hadronic contamination in the broad band beam. This background is clearly limiting the results and one may hope for better information from the tagged photon beams in the not too distant future. 


\section{LESSONS}

What do we learn about heavy quarks from these photoproduction results? First and perhaps most fundamentally, the $\Psi$ and $\Psi^{\prime}$ particles behave like hadrons. In addition to this simple identification, it is possible to study the details of quark, gluon and photon dynamics involving the charmed quark. Furthermore, the fundamental parameters of dynamical theories are ameanable to study; in particular, the mass of the charmed quark. We will come to an example of this mass determination in a moment.

However, it is just in the area of determining the dynamical properties of the interactions where experiments provide the least conclusive results. In the case of the open charm production, there is a discrepancy in the apparent mechanisms as measured at low phot on energy at the CERN SPS and in the higher energy Fermilab broad band beam. In the case of the $\Psi$, the Fermilab broad band photon experiment may not be consistent with the muon experiment observations of equal diffractive and non-diffractive production.6

Three classes of production mechanisms are shown schematically in Figure 7; a) associated production, b) central production and c) diffractive production. In the first of these, the charmed quark produced at the photon materialization vertex interacts directly with quarks in the nucleon. In the case of central production, the interaction with the nucleon may not be vila a quark. In central and diffractive production, one or more of the charmed quarks fuses with a gluon or is scattered with a Pomeron-like mechanism. These last processes cannot provide information directly on quark quark interactions.

In order to determine physically interesting results, we expect to compare measurements to one or another of the processes represented in Figure 7. However, the experimental discrepancies get in the way. The first discrepancy may well be accounted for by the differences in the energies at which the the Charm production was observed. Figure 8 shows the $\Psi$ production cross section threshold behavior as observed in experiments. In addition to the appropriate energy for these measurements, other energy scales are indicated on the abcissa. They are scaled by $M^{2}$, the mass squared of the indicated states: It is clear that within the statistics of the actual measurements, the threshold behavior of producing the heavier DD state and heavier still $\Lambda \Lambda_{c}$ states may account for the apparent discrepancies in the production mechanisms. Not only would this explain the behaviors observed, but it may allow us to obtain additional information from the photoproduction by examining behavior at different energies. 
Once descrepancies are resolved, interesting physical quantities can be determined. As an example, the photoproduction of $\Psi^{\prime} ' s$ is sensitive to interesting physical parameters. Figure 9 provides the threshold and higher cross sections for various charmed quarkmass values in the photon gluon fusion model 7 of Figure 7c. As another example, Figure 10 gives the $p_{t} 2$ distribution of $D^{*} ' s$ in the CFI data and compares it to the predictions of the photon gluon fusion model for three different values of $\vec{c} \vec{c}$ primordial $p_{t}$. Additional information required to achieve the agreement is a soft gluon distribution function of the form:

$$
F_{g}=\frac{(1-x)^{5}}{x}
$$

and a dressing. function of the form:

$$
D_{C}(z)=\exp (-5.5 z)
$$

Clearly, with so much theoretical input, no single experimental result will provide a conclusive measurement of parameters. However, we may hope that a series of measurements will result in a coherent picture.

\section{SCALING FOR TRUTH AND BEAUTY}

The gluon fusion model of the last section can also be used to predict upsilon and open beauty cross sections. The parameters in the theory are crucial for predicting the threshold and asymptotic production levels. However, the threshold behavior can also be predicted by simply scaling the $\Psi$ production threshold behavior measured by previous experiments. In Figure 8, the solid curve is the scaled prediction for bottomonium in the gluon fusion model of Reference 8 . The curve is drawn by adjusting the magnitude of the cross section and scaling the energy by the square of the mass produced in the interaction. As can be seen from the curve and lower scale, Truth lies somewhere in the future for photoproduction. 


\section{ACKN OWLEDGEMENTS}

The data presented in this talk are due to the Columbia-Fermilab-rlinois collaboration and " the Berkeley-Fermilab-princeton collaboration. I am especially indebted to Joel Butler, John Cumalat, Irwin Gaines and Marshall Mugge for providing me with the data and discussing its significance with me. Excellent and more detailed discussions of the data are available in References 9, 10, and 11 . I am also indebted to the INFN for partial financial support of my participation in this conference at Erice. 
FOOTNOTES AND REFERENCES

1. M. S. Atiya, S. Holmes, B. Knapp, W. Lee,

W. J. Wisni ewski (Columbia), M. Binkley, C. Bohler,

J. Butler, J. Cumalat, I. Gaines, M. Gormley,

D. Harding, R. L. Loveless, J. Peoples (Fermilab),

P. Avery, P. Callahan, G. Gladding, M. Goodman,

T. O'Halloran, C. Olszewski, J. J. Russel,

A. Wattenberg, J. Wiss (IIlinois).

2. V. Bharadwaj, B. Denby, A. Eisner, R. Kennett, A. Lu,

R. Morrison, D. Summers, S. Yellin, M. Witherell

(University of California, Santa Barbara).

P. Estabrooks, M. Losty, J. Pinfold (Carleton

University), S. Bhadra, A. Duncan, J. Ellíott,

U. Nauenberg (University of Colorado), J. A. Appel,

J. Biel, D. Bintinger, J. Bronstein, P. Mantsch,

T. Nash, K. Stanfield, S. Willis (Fermilab),

G. Kalbfieisch, M. Robertson (Oklahoma), D. Blodgett,

S. Bracker, G. Hartner, R. Kumar, G. Luste, J. Martin,

K. Shahbazian, J. Spalding, C. J. Zorn (Toronto).

3. A. R. Clark, K. J. Johnson, L. T. Kerth, S. C. Loken,

T. W. Markiewicz, P. D. Meyers, W. H. Smith,

M. Strovink, W. A. Wenzel (Berkeley), R. P. Johnson,

C. Moore, M. Mugge, R. E. Shafer (Fermilab),

G. D. Gollin, F. C. Shoemaker, P. Surko (Princeton).

4. G. Gollin, et al., IEEE Trans. Nucl. Sci., 26, 59 (1979).

5. M. Strovink, Proceedings of the 1981 Int. Symp. on Lepton and Photon Interactions at High Energies, Bonn, August $24-29,1981$.

6. In the version of this paper presented at the conference, particular emphasis was placed on the possible discrepancy in the non-diffractive production of $\psi$. If half the $\psi$ production is non-diffractive, it is difficult to understand how so little of it would have survived the Fermilab-I1linois trigger

requirements. Unfortunately, it is impossible to put quantitative 1 imits on this since the data required to study this question was not recorded.

7. A. R. Clark, et al., PRL 43, 187 (1979).

8. L. M. Jones and M. W. Wyld, Jr., Phys. Rev. D, 17, $2332(1978)$.

9. M. Binkley, et al., Phys. Rev. Lett. 48,73 (1982).

10. J. Wiss, AIP Conference Proceedings, Sixth 
International Conference on Experimental Meson Spectroscopy-1980, Brookhaven National Laboratory, New York, p. 257.

11. J. Butler, Baryon 1980, Proceedings of the IVth International Conference on Baryon Resonances, Toronto, Canada, July 14-16, 1980, p. 329.

\section{$* * *$}

Question (L. Montanet)

In the broad band photon experiment, the $D$ signal is enhanced by selecting events with low $\Delta$-values of $D^{*}+D \pi$. Have they tried the same selection to enhance the $\Lambda_{c}$, i.e., using the low $\Delta$-value of $\Sigma c_{c} \Lambda_{c}$ ?

\section{Answer :}

Yes, the Columbia-Fermilab-Illinois group 100 ked for the low- $\Delta$ enhancement for $\Lambda_{c}$ 's. However, they see no dramatic excess of events with $\Delta^{c}$ about $170 \mathrm{Mev}$. They interpret this to indicate that less than half the observed $\Lambda_{c}$ come from the decay of $\Sigma_{c}$.

Question (B. Margolis)

Do you have any infomation on the ratio of photoproductian of $\mathrm{D}^{*}$ to photoproduction of $\mathrm{D}$ mesons?

\section{Answer :}

Using a $3 \sigma$ excess of $660 \pm 230$ events in the $\mathrm{K}^{-} \pi^{+}$mass distribution, correcting for relative efficiencies and removing $D^{\circ}$ 's from $D^{*} ' s$, the CFI group quotes $.4_{-.10}^{+.22}$ for the $D^{*} / D^{\circ}$ ratio in their data. 
Cross sections were computed using a Monte Carlo program TO CALCULATE THE SPECTROMETER ACCEPTANCE,

ASSUMPTIONS:

1. A $1+\cos ^{2} \theta$ DECAY ANGLE DISTRIBUTION CONSISTANT WITH THE DATA,

2. AN EXPONENTIAL DEPENDENCE ON THE FOUR MOMENTUM TRANSFER SQUARED, $t$, WITH A SLOPE B OF $-4 \mathrm{GEV}^{-2}$. VARIATION OF B FROM 60 TO 1 CHANGED OUR ACCEPTANCE BY LESS THAN $20 \%$ AND PRODUCED NO APPRECIABLE $E$ DEPENDENCE.

PROGRAM INCLUDED EFFECTS OF:

1. Beam Size (2" Square),

2. TARGET LENGTH ( $5 \%$ OF INTERACTION).

3. ChAMBer INEFFICIENCIES (2\% PER TRACK).

4. TRIGGER INEFFICIENCIES,

5. ElECTRON BREMSSTRAHLUNG.

6. GEOMETRIC ACCEPTANCE.

YIELDS CORRECTED FOR:

1. Electronics deAd time (17\%),

2. ACCidental muON halo Vetoes (10\%),

3. TRIGGER COUNTER INEFFICIENCIES (3\%),

4. BeTHE-HEITLER BACKGROUND (5\%).

5. BRANCHING RATIOS, 


$$
\begin{aligned}
& \psi^{\prime} \text { RESULTS } \\
& \sigma_{\gamma P} \rightarrow \psi^{\prime}{ }_{P}\left(\psi^{\prime}+e^{+} e^{-}\right)=6.8 \pm 3.4 \mathrm{nb} \\
& 9 \text { EVENTS } \\
& \sigma_{\gamma P} \rightarrow \psi^{\prime}{ }_{P}\left(\psi^{\prime} \rightarrow \mu^{+} \mu^{-}\right)=5.2 \pm 2.8 \mathrm{nb} \\
& 7 \text { EVENTS } \\
& \sigma_{\gamma P \rightarrow \psi^{\prime} P}\left(\psi^{\prime} \rightarrow \psi \pi_{\pi^{+} \pi^{-}}\right)=6.0 \pm 1.5 \mathrm{nb} \\
& 14 \text { EVENTS } \\
& \text { AVERAGE }=6.0 \pm 1.3 \mathrm{nb} \\
& \text { AT AN AVERAGE ENERGY OF } 160 \mathrm{GEV} \\
& \sigma_{\gamma P} \rightarrow \psi^{p} \mathrm{P}=30 \mathrm{nb} \text { AT SAME ENERGY } \\
& \frac{\sigma_{\psi^{\prime} P}}{\sigma_{\psi P}}=.7 \pm .32
\end{aligned}
$$


AVERAGE PROPERTIES OF $\Lambda_{C}$ EVENTS

(SIGNAL/NOISE $\approx 2 / 3$ )

$$
\begin{aligned}
& \left\langle E_{\gamma}\right\rangle=165 \mathrm{GEV} \\
& \langle t\rangle=.48 \mathrm{GEV}^{2} \\
& \left\langle\mathrm{E}_{\mathrm{A}_{\mathrm{C}}}\right\rangle=83 \mathrm{GEV} \\
& \left\langle P_{\perp, \Lambda_{C}}{ }^{2}\right\rangle=.49 \mathrm{GEV}^{2} \\
& \left\langle E_{K_{S}}\right\rangle=40 \mathrm{GEV} \\
& \langle \# \text { PARTICLES }>=5.2 \\
& \text { (CUT 4-7) } \\
& \left\langle E_{\Lambda_{C}} / E_{\gamma}>=0.52\right. \\
& <\text { TOTAL MASS }>=5.1 \mathrm{GEV} \\
& \langle\text { RECOIL MASS }>=2.5 \mathrm{GEV}
\end{aligned}
$$




\section{CHARMED BARYON PROPERTIES}

$$
\begin{aligned}
& M\left(\mathrm{PK}_{\mathrm{S}}\right)=2284 \pm 1 \pm 5 \mathrm{MEV} \\
& \Gamma\left(\mathrm{PK}_{\mathrm{S}}\right)=7.2 \pm 3 \mathrm{MEV} \\
& \# \text { OF EVENTS }=55 / 75 \text { BG }(\sim 6 \sigma,>8 \sigma \text { FROM FIT })
\end{aligned}
$$

PHOTOPRODUCED

BOTH CHARGE STATES

$$
\frac{\mathrm{P}^{+} K_{\mathrm{S}}}{\mathrm{P}^{-} \mathrm{K}_{\mathrm{S}}} \approx 1
$$

LIFETIME: $\quad \gamma C \tau<3 \mathrm{cM}$ (WOULD HAVE SEEN DECAY)

$$
\begin{aligned}
\langle\gamma\rangle \approx 83 / 2.284 & =36 \\
\tau & <3 \times 10^{-12} \mathrm{SEC}
\end{aligned}
$$

CROSS SECTION

$$
\begin{aligned}
& \text { ACCEPTANCE } \approx 4 \% \\
& \sigma\left(\gamma_{N} \rightarrow \Lambda_{c}\right) \times \quad B R\left(\Lambda_{c} \rightarrow P^{\circ}\right)=3.0 \pm 1 \quad n b / N U C L E O N \\
& \text { USING LINEAR A DEPENDENCE } \\
& \sigma\left(\gamma \mathrm{N} \rightarrow \Lambda_{\mathrm{C}}\right)=200 \mathrm{nb} / \mathrm{NUCLEON} \\
& \text { USING } B R=1.5 \%
\end{aligned}
$$


TABLE V

\section{BRANCHING RATE LIMITS \\ $90 \%$ C.L.}

$$
\begin{aligned}
& \frac{\mathrm{BR}\left(\Lambda_{C} \rightarrow \Lambda \pi^{+}\right)}{\mathrm{BR}\left(\Lambda_{\mathrm{C}} \rightarrow \mathrm{PK}^{\circ}\right)}<0.3 \\
& \frac{\mathrm{BR}\left(\Lambda_{\mathrm{C}} \rightarrow \Lambda \pi^{+} \pi^{+} \pi^{-}\right)}{\mathrm{BR}\left(\Lambda_{\mathrm{C}} \rightarrow \mathrm{PK}^{0}\right)}<3.1
\end{aligned}
$$$$
\frac{\mathrm{BR}\left(\Lambda_{\mathrm{C}}+\mathrm{PK}^{-} \pi^{+}\right)}{\mathrm{BR}\left(\Lambda_{\mathrm{C}}+\mathrm{PK}^{0}\right)}<1.4
$$

$$
\frac{\mathrm{BR}\left(\Lambda_{\mathrm{C}}+\mathrm{PK}^{\circ} \pi^{+} \pi^{-}\right)}{\mathrm{BR}\left(\Lambda_{C} \rightarrow \mathrm{PK}^{\circ}\right)}<3.3
$$


Figure 1. Schematic plan view of the tagged photon. spectrometer showing a multiparticle event.

Figure 2. Effective mass distributions showing $\psi$ ' events from the Fermilab-Illinois collaboration.

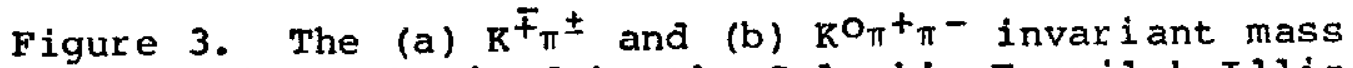
distributions obtained by the Columbia-Fermilab-Illinois Collaboration for combinations within the $D^{* \pm}$ mass difference peak shown in Fig. $4 \mathrm{~b}$.

Figure 4. Mass difference distributions $\left(\Delta \equiv{ }^{M} K_{\pi}-{ }^{M_{K}}\right)$ obtained by the Columbia-Fermilab-Illinois collabo ration for combinations with a $\mathrm{K} \pi$ mass (a) below, (b) straddling, and (c) above the known mass of the $D^{\circ}$. Both charm and anticharm states are included in this plot. The shaded distributions show the appropriately normalized contributions from hadronic contamination in the photon beam.

Figure 5. Proton $\mathrm{K}_{\mathrm{S}}$ effective mass distributions in the search for the $\Lambda_{c}$.

Figure 6. Effective mass distributions for various particle combinations used in the search for charmed baryons. The shaded distributions show the appropriately normal ized contributions from hadronic contamination in the photon beam.

Figure 7. Models of Charm photoproduction.

Figure 8. $\psi$ photoproduction threshold data shown with various erergy scales.

Figure 9. Camparison of the predictions of the photon-gluon fusion model to a fit of the world's $\psi$ photoproduction data for three values of the charmed quark mass.

Figure 10. Comparisons of the $P_{T}^{2}$ distribution of $D^{*}+' s$ to those predicted by a photon-gluon fusion model. 


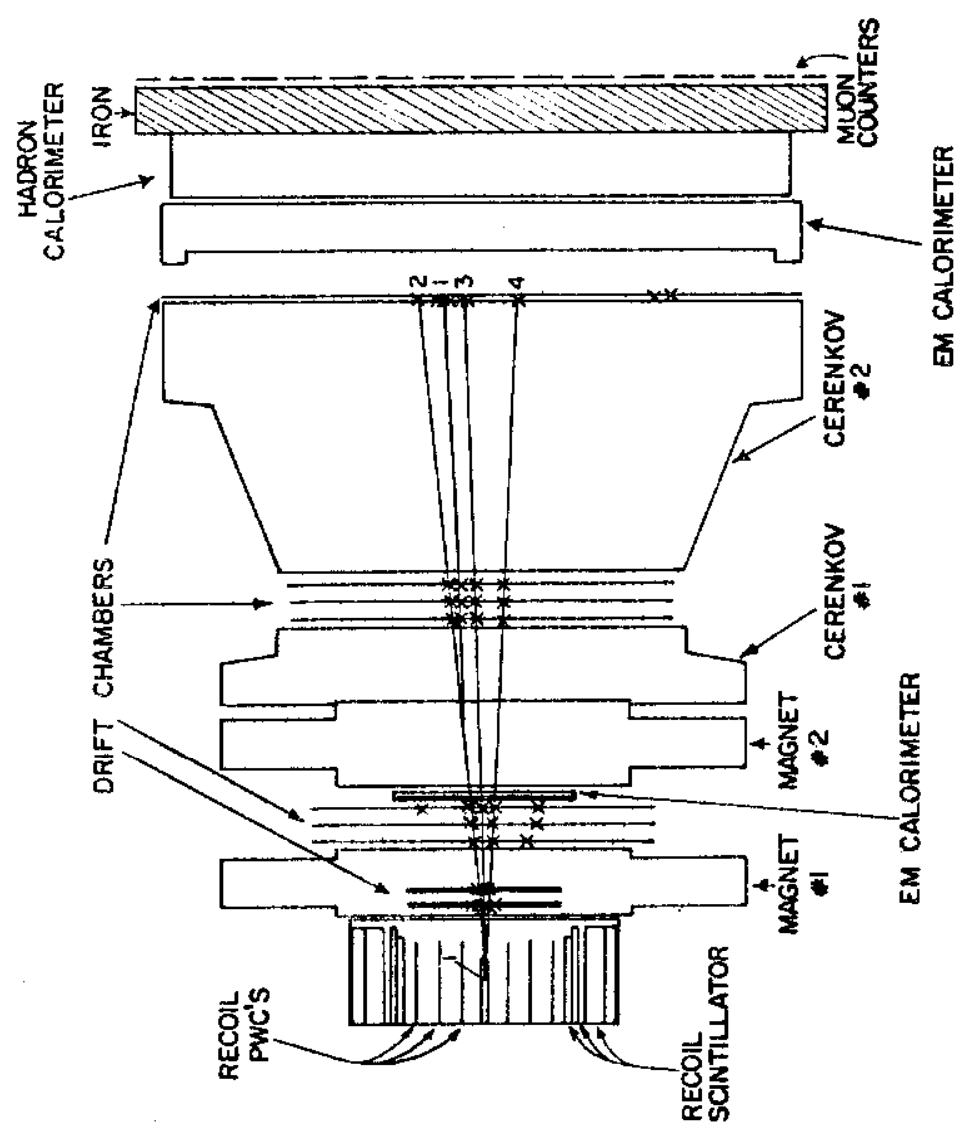



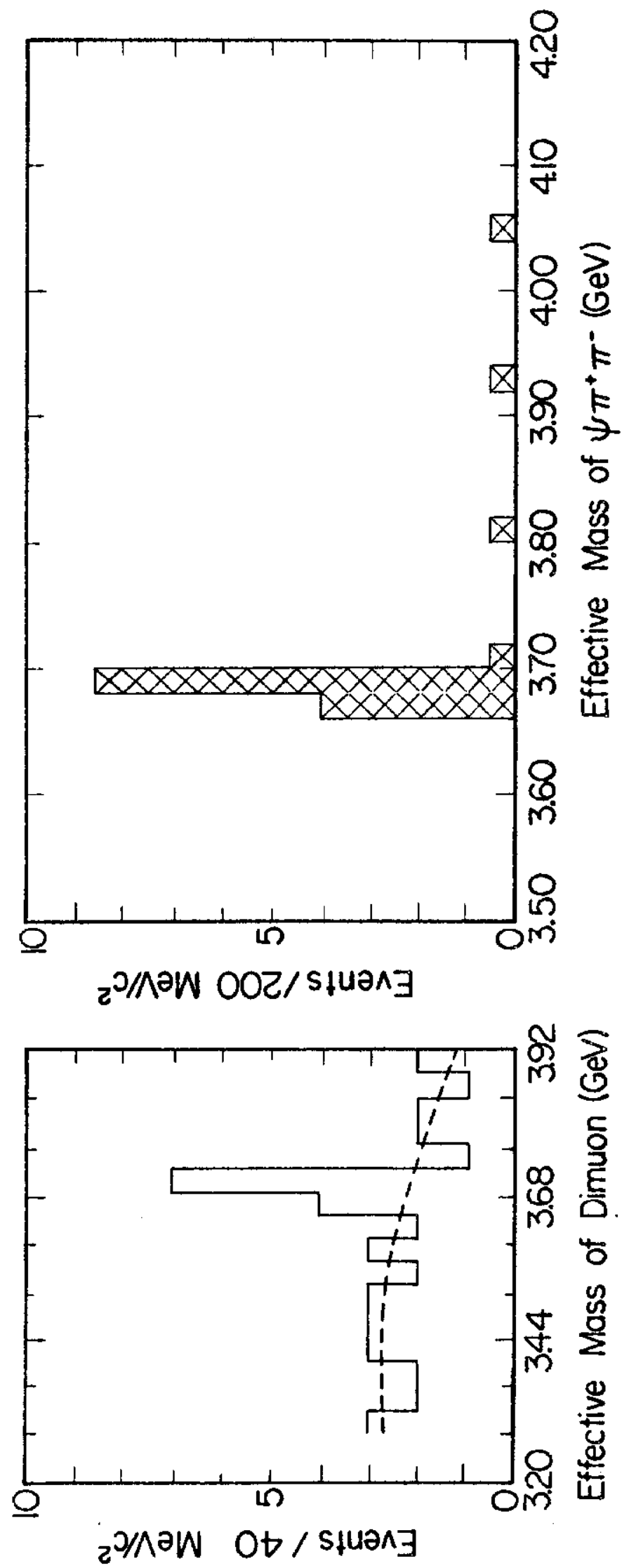


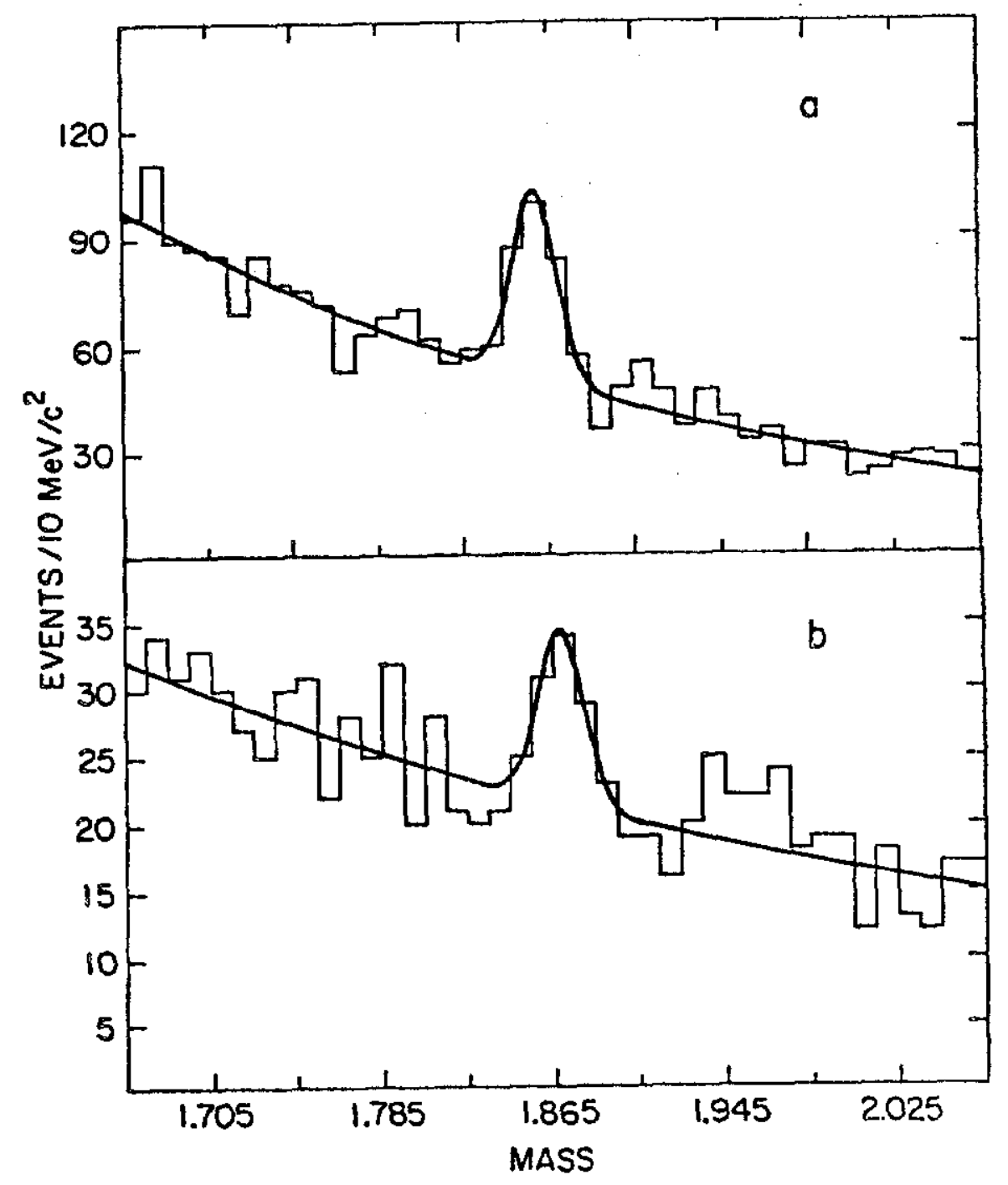

Eigure 3 


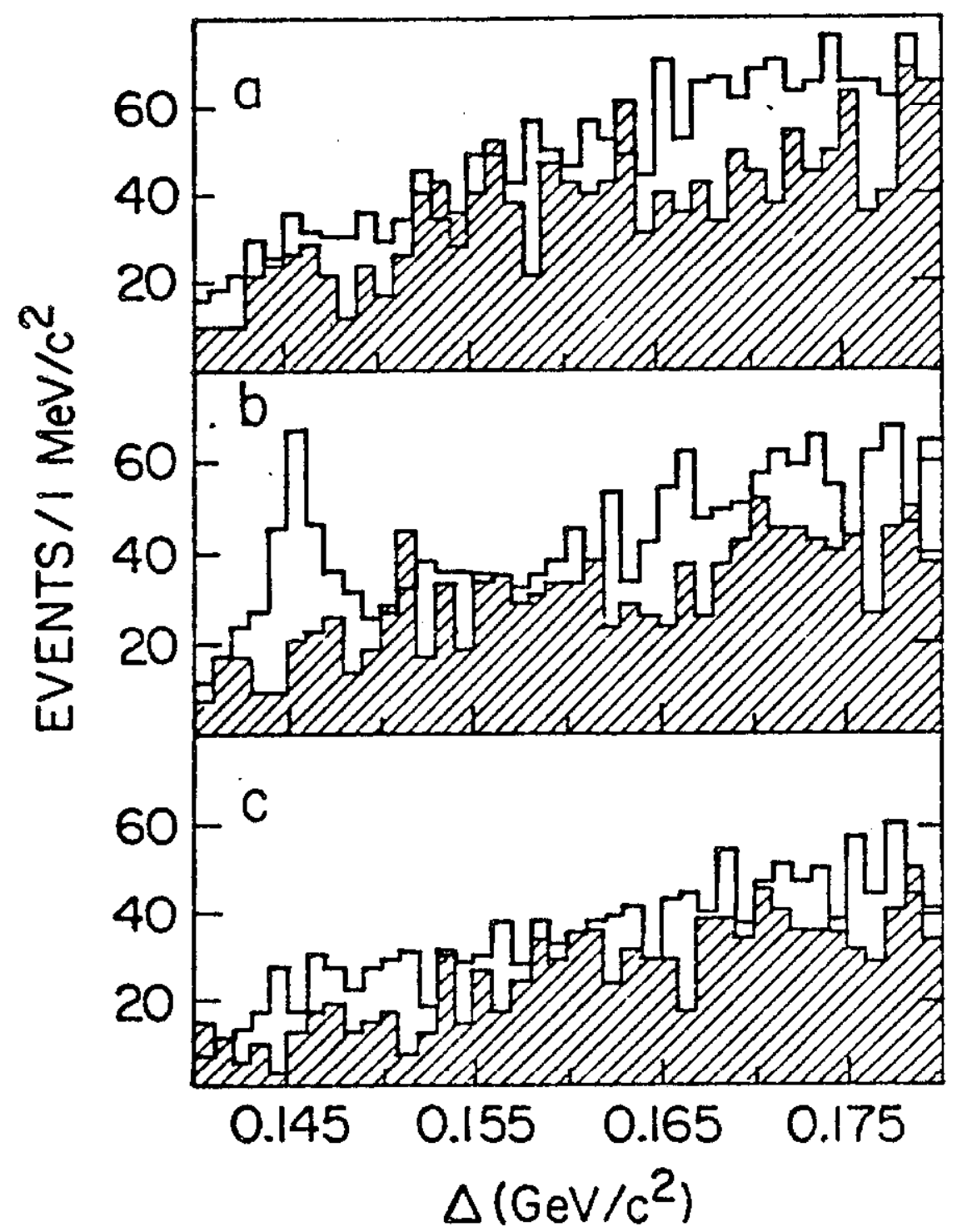

Figure 4 


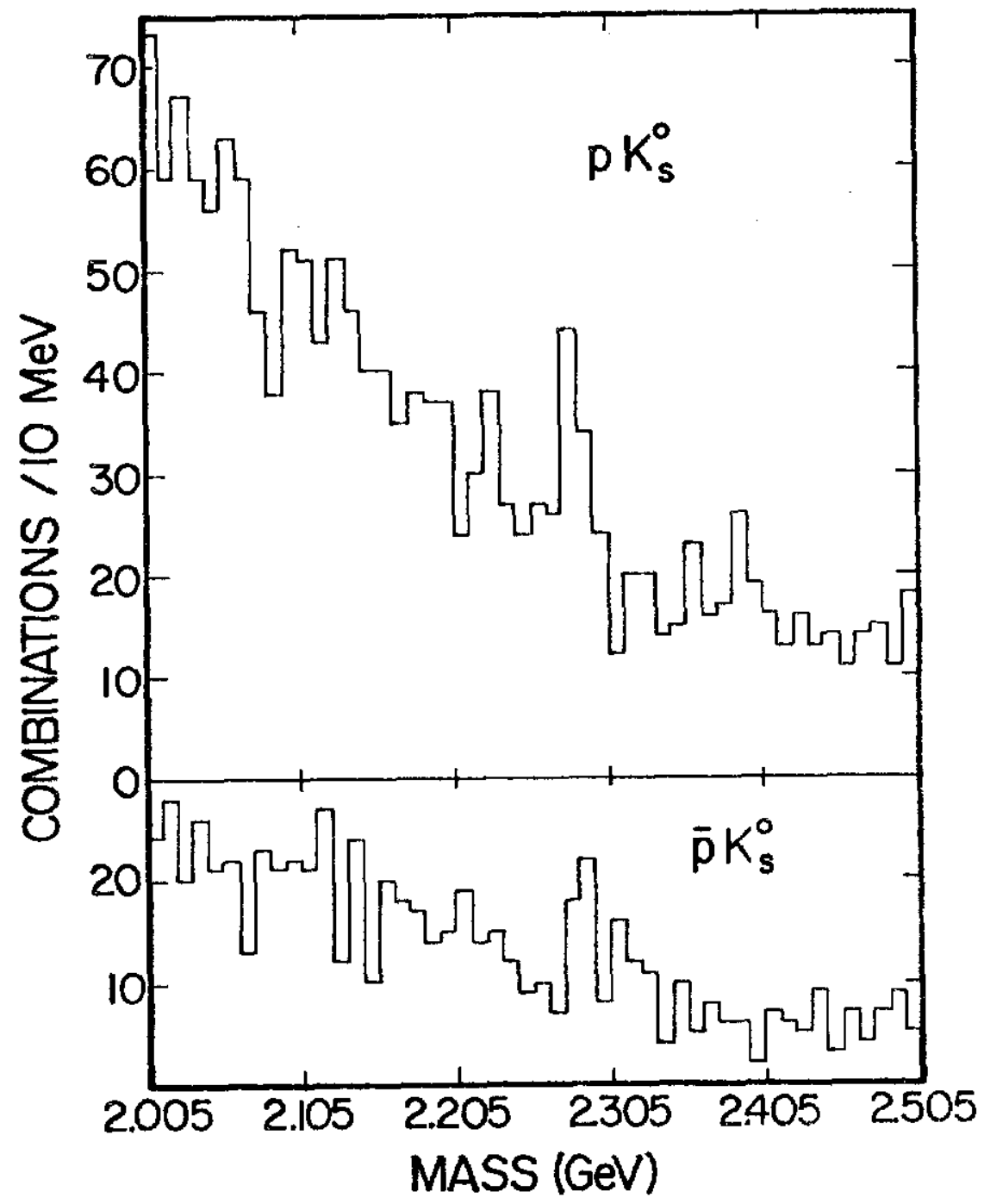

Figure 5 


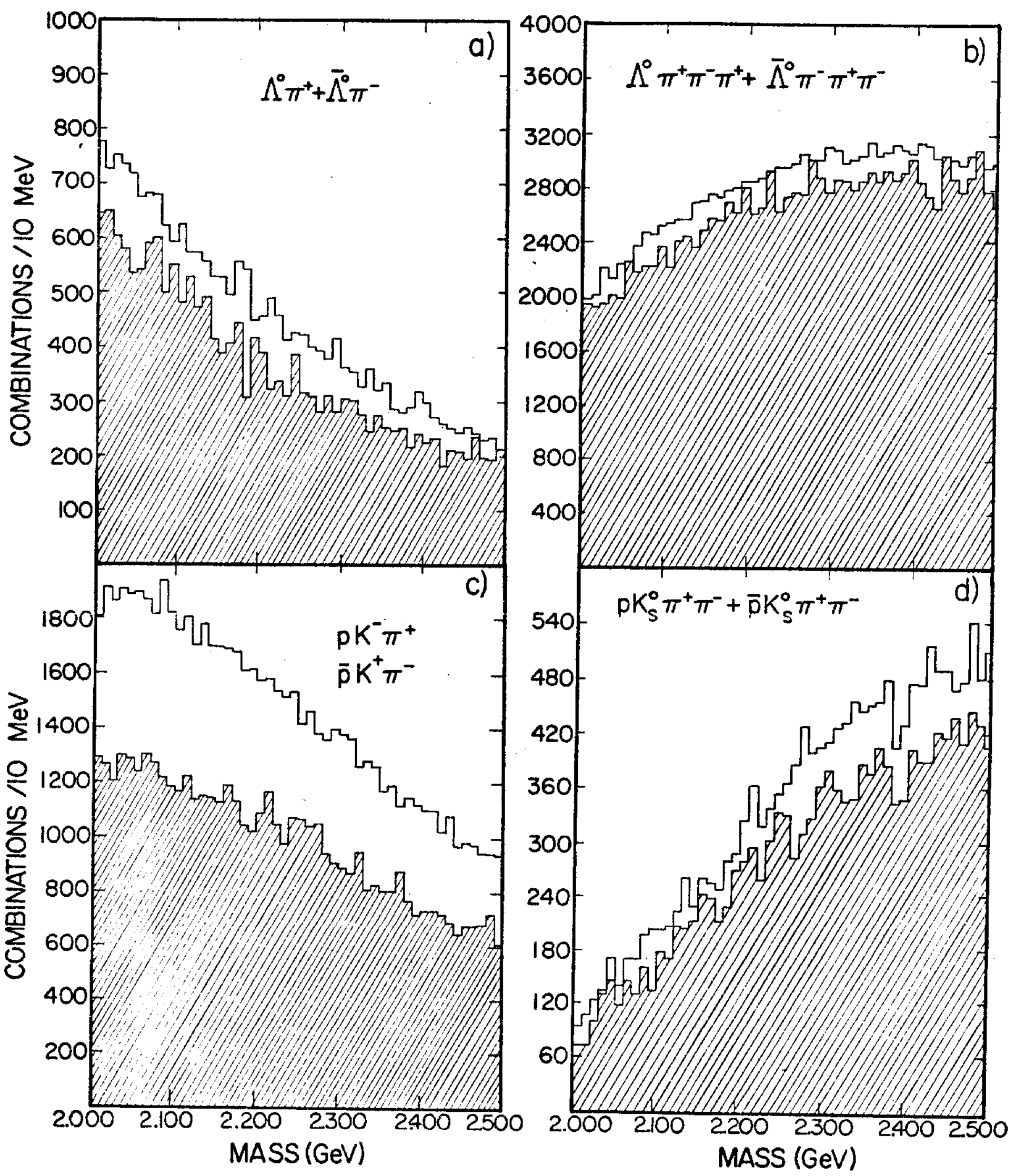




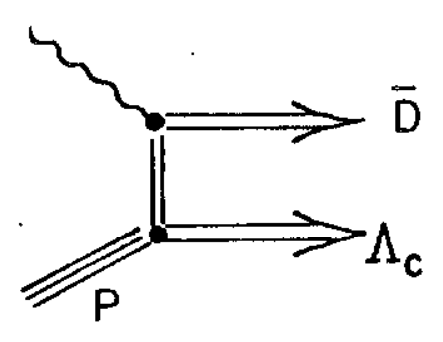

a)

Associated

Production
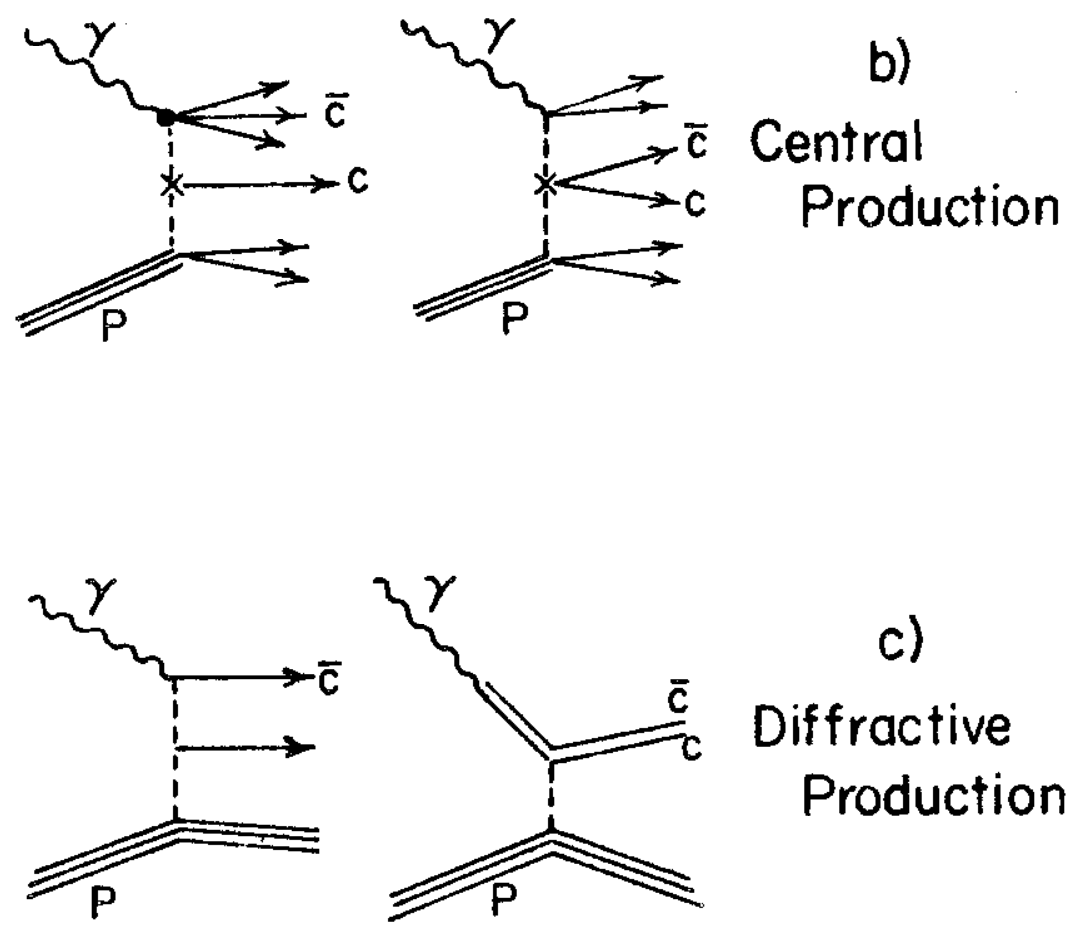

c)

Diffractive

Production 


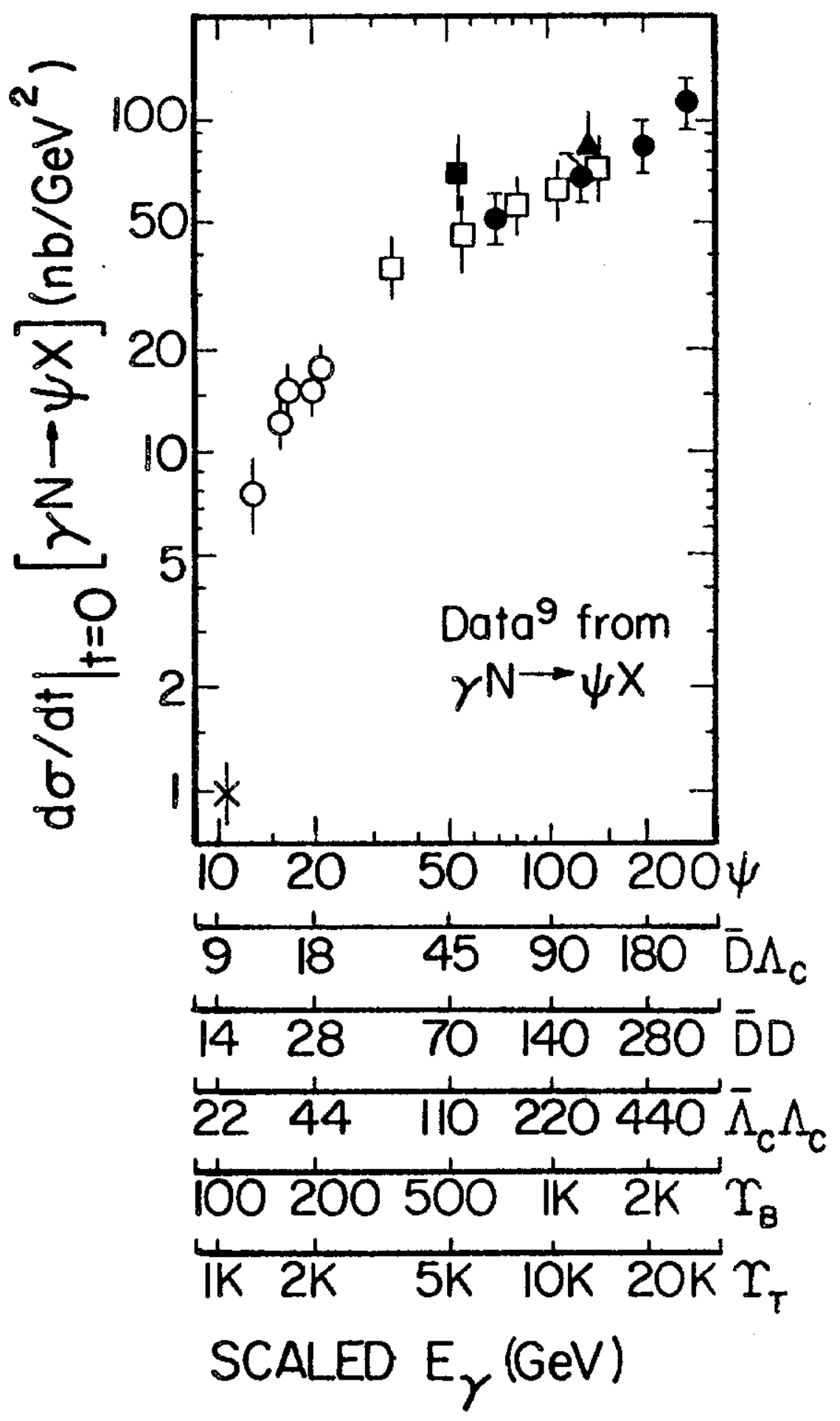

Figure 8 
$a$
0
0
0
0
$0-1$
1

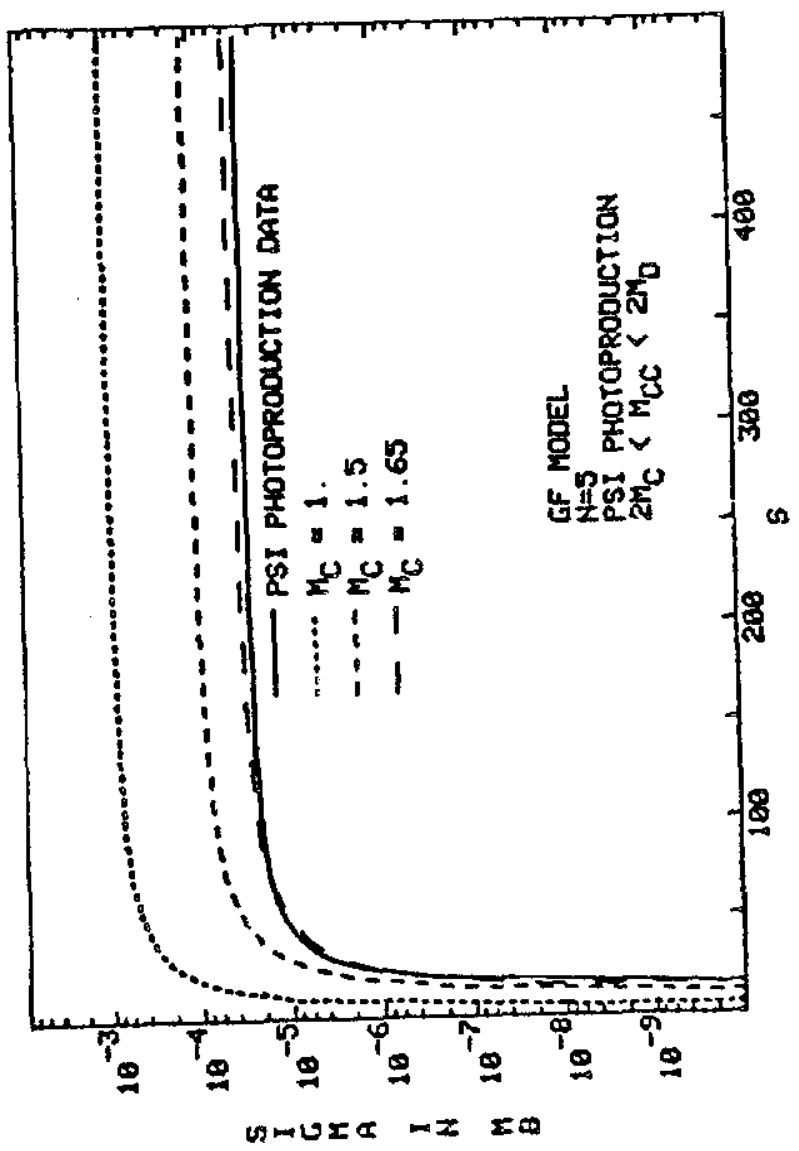



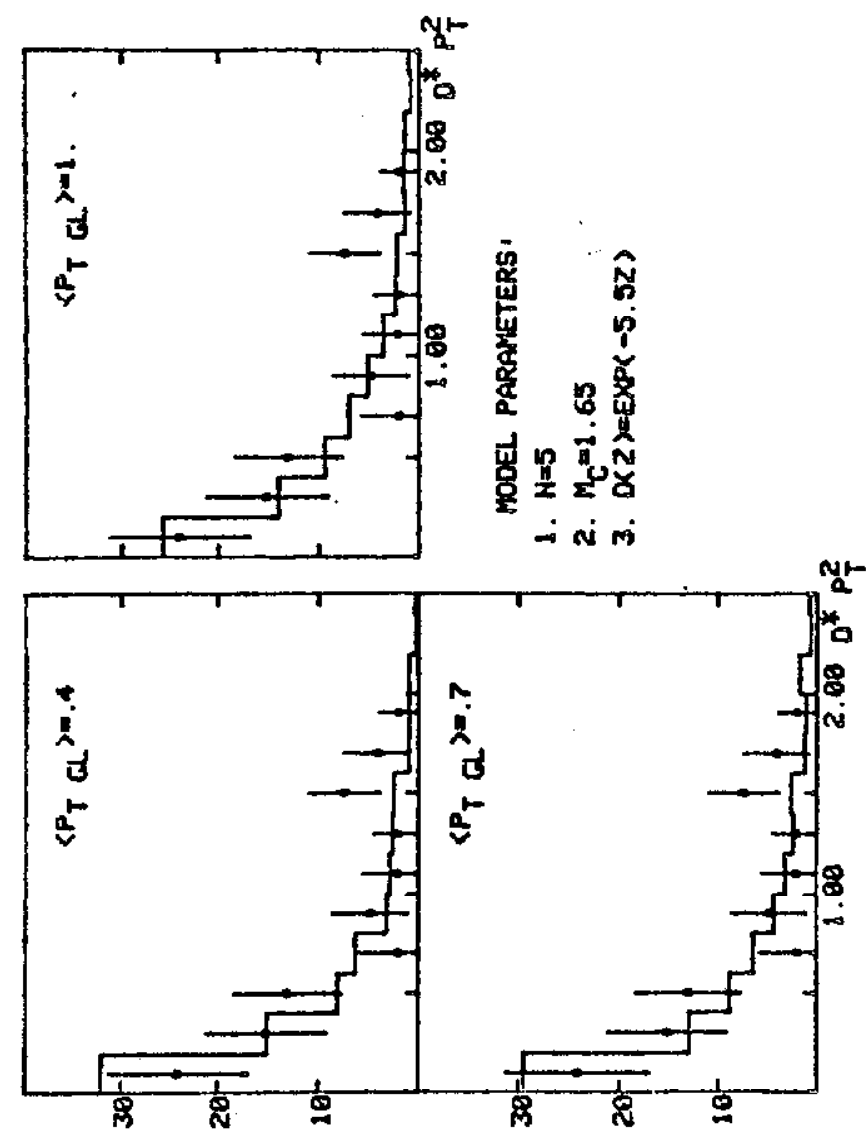